\title{
Electronic evidence and the meaning of "original"
}

\author{
by Stephen Mason
}

Below is the text of part of a lecture I gave on April 17, 2009 under this title at the Tokyo Institute of Technology, Graduate School of Decision Science and Technology, Ookayama-campus, Tokyo, at the invitation of Professor Hironao Kaneko.

W hen I write a haiku, I invariably, but not always, incorporate the three elements into the poem, as expressed by Kenneth Yasuda: “.... in haiku, the poet usually attempts to present a speaking object, around which and in which he has an experience" (Kenneth Yasuda, The Japanese Haiku (Tuttle, 1957, 1991), 41). The three elements that comprise the properties that constitute the experience are the "when", "where" and "what."

I wanted to express the concept of "original" because lawyers are used to dealing with an original document, or an authenticated copy of the original of a document, but are in difficulties when the concept of "original" proves to be elusive in respect of a digital object, whatever form it takes (for example, a photograph in the form of a TIF image, a document published into PDF, or a database). Arguably, there is no concept of an "original" digital object, which means it is necessary to reconsider the conceptual framework, which many lawyers have failed to do thus far. I have argued that the nearest we can get to the concept of an "original" digital object, is to recognize that it is necessary to consider how authentic the digital object is, and this in turn can be reduced to the following:

“(a) The content of the data that a party relies upon has not changed from the moment it was created to the moment it is submitted as evidence.

(b) The data can be proven to be from the purported source.

(c) The technical and organizational evidence demonstrates the integrity of the data is trustworthy, and is therefore considered to be reliable." (Stephen Mason, general editor, Electronic Evidence: Disclosure, Discovery \& Admissibility

(LexisNexis Butterworths, 2007) 4.35).

In the haiku I wrote for another text on electronic evidence (Stephen Mason, general editor, International Electronic Evidence (British Institute of International and Comparative Law, 2008), I wanted to illustrate the difficulty in determining what an original might be by looking at nature (unlike many Japanese haiku, I tend to give a title to a haiku, and in this case, I think it necessary):

\section{Original}

Rain droplets land, merge,

divide on petals of the

Somei Yoshino.

Water is an ideal mechanism to demonstrate this conundrum (a problem wholly of human making, because documents do not exist in nature), mainly because of the physical properties of water: that is, water can exist, amongst other forms, as a mass (the ocean), or in the form of a droplet of rain falling from a cloud. In addition, water can be subject to rapid changes in shape and size when it forms part of a waterfall, for instance. One moment it can be a minute particle forming part of the mist, the next it can fall and form part of a lake.

This haiku might be considered a double haiku, consisting of two layers: the explicit description and discussion of a physical occurrence in nature with water and the petals of cherry blossom, and a secondary examination of digital evidence. This is discussed in more detail below.

\section{WHEN}

There are two layers to the "when" in this haiku. First, the physical world of humans and nature as the earth moves out of winter, and the cycle of renewal begins again. We are in spring - there is no need to imply, suggest or associate the reference to spring from the reference to the petals on the cherry tree (which, in itself, is not a seasonal word, such as "haze" for instance, and the direct words "cherry blossom" are avoided as a categorical seasonal word), because in Japan the blossom is spring, because the cherry blossom flowers in spring; indeed, spring is incorporated into the cherry blossom. There is another reason for referring to the petal, and that is because the petals are the first to appear on the tree, then the petals 
give way to the leaves, and the tree continues with its cycle. The petals (forming flowers) are, arguably, the first part of the cycle, and the most colourful and dramatic (it will be wrong to say the best part, because the tree in leaf continues to live, and is not any worse for no longer having blossom). Also, the reference to the spring is by means of a living plant (not an abstract reference such as "spring"), and the associations with that plant, "petals", which also emphasises the importance of the cherry blossom in the national life of Japan. In this respect, my aim was to refer to the physical emergence of the petal, giving the petal a central role in the haiku by reference to the "when", and well as the surface upon which the droplets of rain merge and split, linking what happens to the water to what can (and does) happen to digital objects. Finally, the petals, with such a short life, are a metaphor for the ephemeral nature of life - it is also a metaphor for the apparent temporary existence of digital objects.

A light fall of rain completes the picture of the physical world in spring and the celebration of the cherry blossom. The winter is over, but we are reminded that showers of rain will persist until the summer takes hold. Naturally, rain descends in the summer, but it is not a surprise that it should rain during the spring. From the point of view of describing the physical world within the haiku, the aim is to bring these associations together, and for them to be taken up by the reader or listener in such a way that each person brings their own experience of spring to the haiku.

The second layer is reference to the digital world. Although the digital world has been with us for a few decades now, nevertheless it is only recently that the effect of the digital world, with the networking capabilities of the world wide web and the internet, has begun to affect everyone, including the law. For many lawyers, digital evidence can be considered similar to the revealing of the blossom in spring for the first time. The spring represents the beginning of the cycle, and the digital world, which exists in parallel with the physical world, together with evidence in digital format, is only in its infancy. The vast majority of lawyers have failed to appreciate this, and universities and law schools, with rare exceptions, also ignore the new reality - at the ultimate cost to the client.

\section{WHERE}

It must be fair to say that the geographical location could be anywhere that the Somei Yoshino (Yoshino Cherry, prunus $x$ yedoensis) grows naturally, other than in Japan. But the purpose of the reference to the Somei Yoshino is to refer to the ubiquitous nature of cherry blossom as a symbol of Japan. In addition, the very name of the tree indicates where it was first thought to be cultivated; moreover, it is also the most numerous cherry tree in Japan. Even if this does not convince the reader or listener to accept that the "where" is in Japan, given the absence of an identified location and the use of the Japanese word "Somei Yoshino", it remains possible for the reader or listener to make their personal association with a time and place from their memory. In this respect, it might not be necessary for the "where" to be included in order to experience a personal response to the haiku, although it is also possible to experience the haiku in a secondary way, that is if the reader or listener has never been to Japan to witness the cherry blossom season, they may extend their imagination to wonder how the cherry blossom might be celebrated in Japan.

The ambiguity about "where" highlights the second layer, the reference to the digital world. A document might be created on board an aeroplane flying between two continents. During the flight, if permitted, the document could then be sent to any number of recipients in any number of countries. In this respect, the geographical location of a digital object may not matter - unless it becomes highly relevant for the purpose of legal proceedings, and then the "where" and the time the digital document was created may be of crucial importance. The evidence of the text in the haiku tends to support the proposition that the "where" is Japan, which in itself is hardly geographically specific, although the evidence is not conclusive. Evidence in digital format has the same characteristics. A digital evidence specialist might be able to offer an initial generalization as to the origin of a digital object, but they will not be certain until they conduct further investigations and reach a reasoned conclusion based on the evidence they have found.

\section{WHAT}

The reference to water, as already referred to, enables the reader or listener to reflect on their own experiences of rain, but also to extend their experience by imagining how close they can be to a petal upon which droplets of water have fallen. The aim is to bring the reader or listener into the centre of the haiku, and make what happens in the haiku an event that they have witnessed. This is just water: droplets of water, yet the reader or listener follows the tiny bead of water on the petal, recognizes that a gentle breeze can make them move on the petal, some of them might merge together, then a sudden flip of wind causes the bigger drop to fall into the air and split into smaller droplets as it hits a surface in its descent to earth, on to another surface, or falls into a puddle. The insight is to recognize that water, which is fundamental to life, can be viewed in a way other than as an essential part of life, or a nuisance when we are outside without a coat or umbrella when the rain falls unexpectedly. He we understand one of the properties of a liquid - water - in a different way. Water and one of its attributes reflects "originality" in nature, and each reader or listener in turn experiences an original response to the haiku that is unique to them, but everybody that reads or listens to the haiku shares a similar experience.

The personal memory and experiences that lie beyond the physical changes to the droplets of water accompany 
the secondary theme in the haiku, that of digital objects. Not everybody living on this earth has experienced the digital world yet, but large numbers of people have some knowledge of the continually expanding digital world. For instance, a photograph taken with an analogue camera (that is, a camera with a film) can only remain a single object. It cannot be merged into other photographs, and split off again. It remains a physical object. A photograph taken with a digital camera differs markedly. The digital object, made up of a series of zeros and the number one, can be, and frequently is, manipulated and altered (especially in fashion magazines and for advertisements). Things can be taken out and put in to the image, in the same way the water droplets can merge and form a single, larger droplet. The new, manipulated digital image can also be divided back into its constituent parts.

Herein lies the interesting point: when three droplets of water fuse and then separate into three droplets, it is to be questioned whether the three droplets that merge from the bigger droplet were the identical droplets that existed before they merged. In the same way, consider a digital object that has been manipulated and added to, and the process is then reversed. The original object that was used remains (unless it was never saved independently, and the changes made to the image were saved in the original file), but another object, with the identical image (or near identical, depending on the system software and application software) now exists. Conceptually, it is possible to argue that the two digital images are different: one is the original, the other a copy of the original that was manipulated and returned to its original state (whatever "original" means). But both images are identical, apart from some additional meta data that might, or might not be conclusive. However, it is apparent that the images, if viewed together, are identical - will be identical, and the viewer will not be able to determine which is the original, and which image was manipulated. In this respect, the digital images are no different from the droplets of rain that fall, merge, then divide: there is no telling whether the droplets that split are identical to the droplets that came together to form the larger droplet.

\section{CONCLUDING REMARKS}

My ultimate aim in writing this haiku was to achieve what Kenneth Yasuda described as to “... open up greater understanding of what a man is, of what his relationship to the world is" (for "man" please also read "woman"). In this respect, I hope the haiku, on the superficial level, heightens the physical experience of observing nature for the reader or listener for the few seconds it takes to read. For the more perceptive reader or listener, I hope to have extended their experience beyond the pleasures that nature affords, into the realm of a world wholly created by human beings, offering a minuscule insight into the digital world.

\section{Stephen Mason}

(C) Stephen Mason, 2009 